\title{
Colonic perforation in the first few hours of life associated with rhizomelic chondrodysplasia punctata
}

\begin{abstract}
Rhizomelic chondrodysplasia punctata (RCP), a rare autosomal recessive disease characterized by a disorder of peroxisome metabolism, has been shown to affect multiple organ systems. A neonate presenting with a colonic perforation in the first few hours of life was subsequently diagnosed with RCP. A literature search revealed no previous reports of intestinal perforation associated with RCP. Intestinal perforation should be added to the list of medical complications associated with RCP.
\end{abstract}

Keywords Rhizomelic chondrodysplasia punctata Colon perforation

\section{Introduction}

Rhizomelic chondrodysplasia punctata $(\mathrm{RCP})$ is a rare autosomal recessive disease of peroxisome metabolism. RCP affects about 1 in 100,000 individuals [1]. Spranger et al. first described the clinical diagnostic criteria of severe and symmetrically shortened proximal long bones (rhizomelia), bilateral cataracts, and severe growth and psychomotor delays $[2,3]$. Evidence of congenital contractures, typical dysmorphic facial characteristics, and radiographic evidence of stippled epiphyses and vertebral coronal clefts can also be diagnostic [4]. White et al. recently compiled an excellent review of RCP based on clinical experience, family questionnaires, and a review of the literature [4]. Their results summarize and synthesize data and literature reports that were sporadic and disjointed.

T. Fairbanks · S. Emil $(\bowtie)$

Division of Pediatric Surgery, University of California,

Irvine Schoole of Medicine, Bldg. 53, Rt. 81,

101 The City Drive, Orange, CA 92868, USA

E-mail: semil@uci.edu

Tel.: + 1-714-4568581

Fax: + 1-714-4568931
Survival of patients with RCP is reported to be $90 \%$ at 1 year of age and $50 \%$ at 6 years [4]. Infants and children with RCP grow exceedingly slowly. Profound mental retardation is usually seen, and seizures are also common. Cataracts are present at birth in nearly all infants. Many patients experience serious pulmonary issues, with two-thirds having respiratory distress after birth. Cardiovascular defects are rare but may be present.

Skeletal abnormalities are a hallmark of the disease, with severe joint contractures predominating. Lesions that can occur and that require surgical intervention include diaphragmatic hernia, cleft palate, hypospadias, and cryptorchidism. R.M. Pauli, the senior author of the review by White et al. [4], was aware of only one case of intestinal perforation, which resulted from a gastrostomy tube placement in a patient with RCP (personal communication). No spontaneous perforations have been previously reported with this disease.

\section{Case report}

A newborn male was delivered by cesarean section secondary to breech presentation at 33 weeks' gestation. The mother was visiting from a neighboring state when she went into preterm labor. She received two courses of betamethasone and penicillin. The infant's birth weight was $2,026 \mathrm{~g}$. Apgar scores were 7 and 8 at 1 and $5 \mathrm{~min}$, respectively. Following delivery, the infant was noted to have some respiratory distress with grunting and mild retractions. He was admitted to the neonatal intensive care unit and treated with supplemental oxygen and a brief course of continuous positive airway pressure.

About $10 \mathrm{~h}$ after delivery, the infant's clinical condition worsened. He had a brief apneic episode followed by a worsening of his respiratory distress. Abdominal distention developed; meconium had not been passed. He remained hemodynamically stable with good urine output. Radiographs of the chest and abdomen were obtained, which showed gross bone abnormalities and 
pneumoperitoneum overlying the liver on the cross-table view. An orogastric tube was placed, and the pediatric surgery service was consulted.

Physical examination of the abdomen revealed moderate distention, firmness in both upper quadrants, and no tenderness or signs of peritonitis. The anal canal was narrowed, and a cotton-tipped swab could not be advanced past $2 \mathrm{~cm}$ from the anal verge. A complete blood count was unremarkable. A water-soluble contrast enema showed a contained perforation at the hepatic flexure of the colon proximal to a microcolon (Fig. 1).

At laparotomy, a colonic perforation at the hepatic flexure, contained by the liver and anterior abdominal wall, was found. The right colon appeared inflamed, and the distal ileum was plugged with thick meconium. A right hemicolectomy was performed, with an ileostomy and mucus fistula. Pathologic examination was nonspecific, showing ischemic changes with some evidence of adhesions. Ganglion cells were present.

The genetics service was consulted, and the diagnosis of RCP was made based on clinical findings, bone imaging (Fig. 2), and red cell plasmalogen levels. Genetic markers for cystic fibrosis (CF) were sent. One DNA mutation for CF was found. The karyotype was normal $46 \mathrm{XY}$.

Bowel function was slow to return. Imaging with small bowel follow-through did not reveal any further mechanical obstruction, but motility was significantly slowed. Stenosis of the ileostomy occurred and required revision. Goal enteral feeds were eventually achieved over approximately 10 weeks but were given partially by a nasogastric tube because of poor suckling.

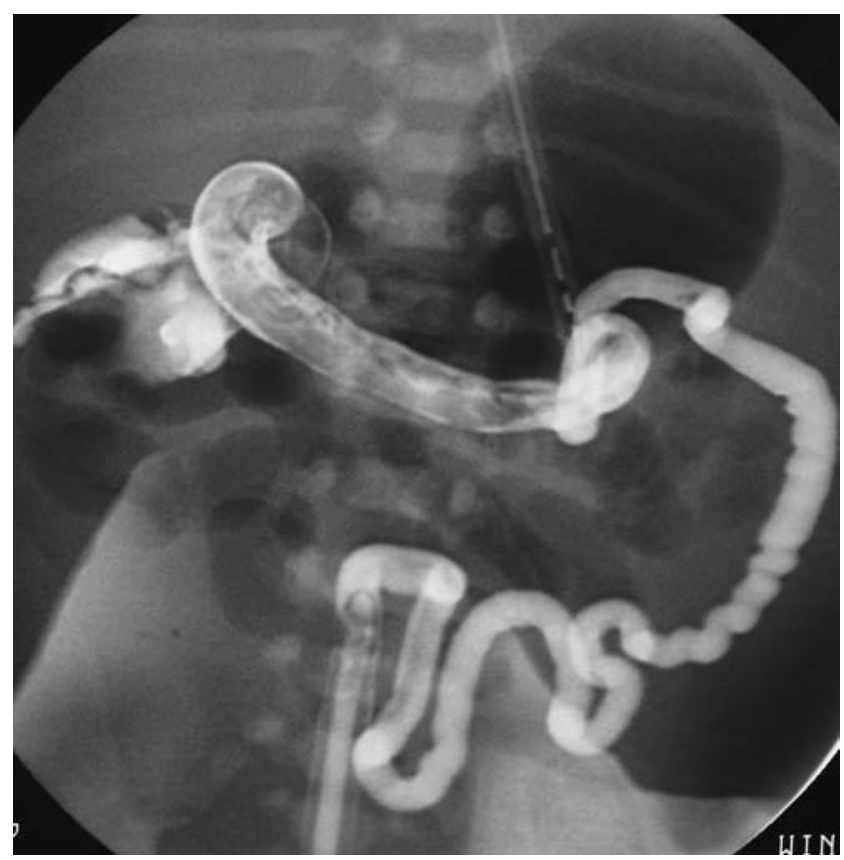

Fig. 1 Water-soluble contrast enema demonstrating a microcolon, a large meconium plug in the transverse colon, and a contained perforation at the hepatic flexure of the colon

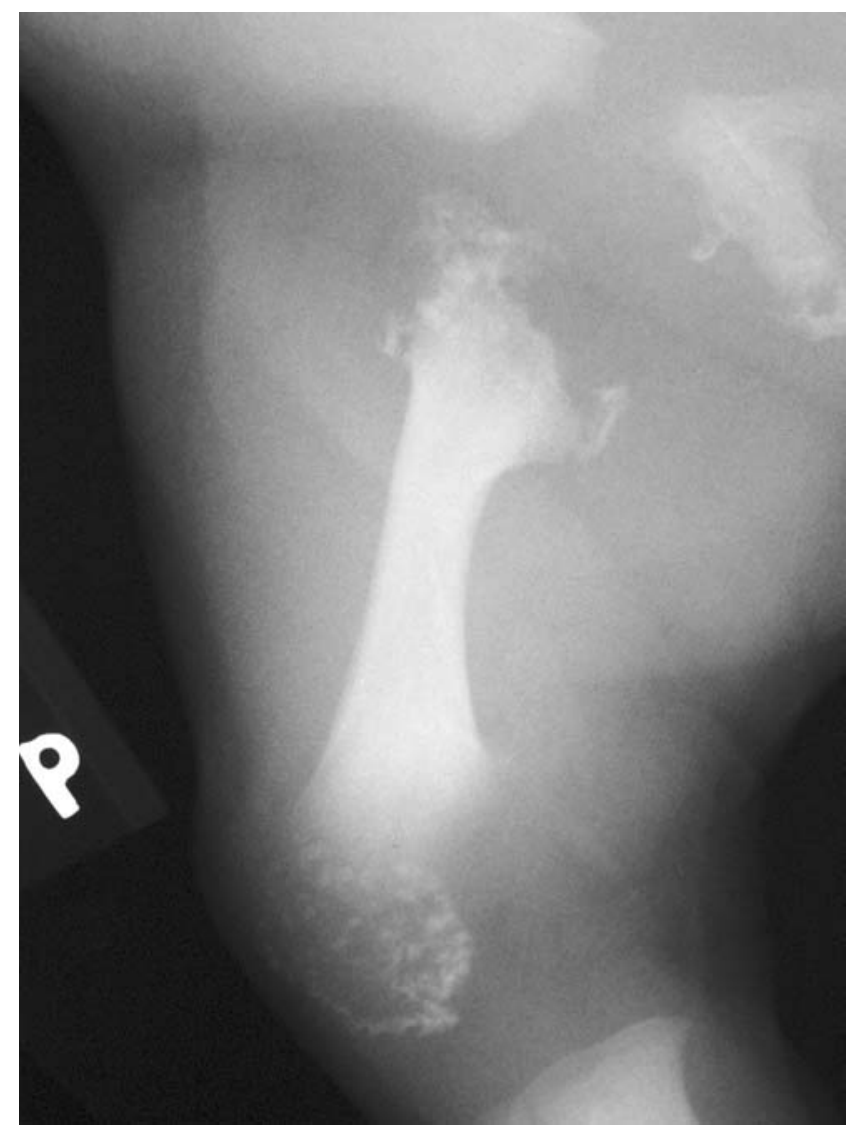

Fig. 2 Right femur radiograph demonstrating shortening of longitudinal growth, broad metaphyses, and stippling of the calcific epiphyses. All are typical features of RCP

The infant was discharged to home in a neighboring state where his medical care continued. He succumbed to severe sepsis secondary to pneumonia at age 6 months.

\section{Discussion}

$\mathrm{RCP}$ is an autosomal recessive disorder of peroxisomal metabolism caused by genetically heterogeneous mutations of the PEX7 gene located on chromosome 6q22-24 [5-7]. This gene is important for the synthesis of specific peroxisomal membrane proteins. The biochemical and metabolic results of inactivation of the $P E X 7$ gene have been well reported for their phenotypic presentation in the tissues of the bone, eye, and brain, among others. Phenotypic abnormalities of the intestinal tract have thus far not been reported.

This report represents the first known case of spontaneous intestinal perforation in a neonate with RCP. RCP is a severe disease that has been well documented to have a profound impact on the respiratory, cardiovascular, nervous, ophthalmologic, otologic, dental, and musculoskeletal systems. Although the long-term survival of these patients has improved over the years, it is clear that they have a short lifespan with severe growth 
and developmental delays. It is logical that an abnormal peroxisomal membrane protein synthesis, which globally affects multiple organ systems, would impact intestinal development, regeneration, and repair.

The exact pathophysiologic process that led to intestinal perforation in this patient is unclear. The perforation likely occurred in the late prenatal period, shortly before delivery. This would explain the containment of the intraperitoneal perforation by the liver and abdominal wall. The absence of an associated atresia makes a major vascular accident or etiology unlikely. The absence of calcifications indicates that the perforation did not take place early in gestation. The perforation is unlikely to have been a spontaneous intestinal perforation similar to that seen in severely premature babies with very low birth weights. Spontaneous intestinal perforations typically occur in the ileum, are strongly associated with indomethacin (which was not used in this baby), and are unlikely in the first few hours of life. The colon may have perforated proximal to the functional obstruction imposed by the microcolon and meconium plugging. The intestinal dysmotility observed in this patient points to an insult affecting the entire gut.

The presence of thick meconium plugging the distal ileum causing a functional obstruction is suspicious of $\mathrm{CF}$ or a CF-like syndrome. It is also interesting to note that the patient had a single DNA mutation for the CF gene. A single DNA point mutation should only be sufficient to cause a heterozygous state. Heterozygous invalidation of the $\mathrm{CF}$ gene should not cause meconium plugging. It may be that the $\mathrm{CF}$ gene pathway is involved with peroxisome metabolism in the gut such that the invalidation of one pathway exacerbates problems with the other. This may also help explain why many infants with RCP, including the one reported here, have respiratory difficulties that eventually lead to their demise.

This report of intestinal perforation presenting in the first few hours of life in a patient with RCP may allude to some role of the PEX7 gene in gastrointestinal development. It is also possible that this patient's presentation was the result of a CF-like syndrome. Intestinal perforation should be added to the list of potential pathologies in patients with RCP.

\section{References}

1. Stoll C, Dott B, Roth MP et al (1989) Birth prevalence rates of skeletal dysplasias. Clin Genet 35:88-92

2. Spranger JW, Bidder U, Voelz C (1971) Chondrodysplasia punctata (Chondrodystrophia calcificans) II. The rhizomelic type. Fortschr Geb Rontgenstr Nuklearmed 114:327-335

3. Spranger JW, Opitz JM, Bidder U (1971) Heterogeneity of Chondrodysplasia punctata. Humangenetik 11:190-212

4. White AL, Modaff P, Holland-Morris F, Pauli RM (2003) Natural history of rhizomelic chondrodysplasia punctata. Am J Med Genet 118A:332-342

5. Braverman N, Steel G, Lin P et al (2000) PEX7 gene structure, alternative transcripts, and evidence for a founder haplotype for the frequent RCDP allele, L292ter. Genomics 63:181-192

6. de Vet EC, Ijlst L, Oostheim W et al (1999) Ether lipid biosynthesis: alkyl-dihydroxyacetonephosphate synthase protein deficiency leads to reduced dihydroxyacetonephosphate acyltransferase activities. J Lipid Res 40:1998-2003

7. Ofman R, Hettema EH, Hogenhout EM et al (1998) Acyl-CoA: dihydroxy-acetonephosphate acyltransferase: cloning of the human cDNA and resolution of the molecular basis in rhizomelic chondrodysplasia punctata type 2 . Hum Mol Genet 7:847-853 